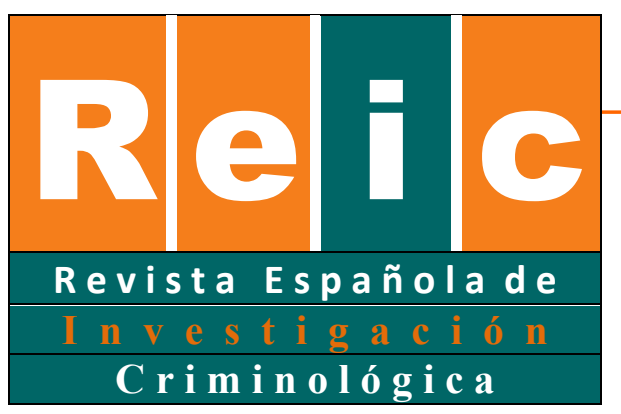

Panyella-Carbó, Agustina \& Martin-Fumadó

\title{
Sumisión química versus vulnerabilidad química: análisis criminológico de los delitos sexuales facilitados mediante el uso de sustancias psicoactivas a partir de una muestra de sentencias
}

Proactive versus opportunistic Drug-Facilitated Sexual Assault: criminological analysis of sexual crimes facilitated by the use of psychoactive substances from a sample of court decisions

Maria Neus Panyella-Carbó y José R. Agustina (iD) 1

Universitat Internacional de Catalunya

Carles Martin-Fumadó (iD

Universitat Internacional de Catalunya

Cátedra de Responsabilidad Profesional Médica y Medicina Legal, Departamento de Psiquiatría y Medicina Legal, Universitat Autònoma de Barcelona

\section{RESUMEN}

El presente artículo analiza la fenomenología delictiva de los delitos de abuso sexual cometidos mediante el uso de sustancias psicoactivas. Se llevó a cabo un análisis descriptivo de una muestra de sentencias, como resultado de procedimientos penales seguidos en España entre 1999 y 2016, examinándose distintas variables disponibles sobre el hecho delictivo, la víctima y el agresor. De la muestra analizada se observa que en la mayoría de casos (76\%) el agresor se aprovechó de una situación de "vulnerabilidad química", siendo la propia víctima la que había consumido voluntariamente sustancias psicoactivas. Solo en el $24 \%$ de casos

${ }^{1}$ La correspondencia debe dirigirse a Maria Neus Panyella-Carbó (mnpanyella@uic.es). Facultat de Dret, Universitat Internacional de Catalunya, C/ Immaculada, 22 - 08017 Barcelona, España.

Revista Española de Investigación Criminológica

Artículo 5, Número 17 (2019)

https://doi.org/10.46381/reic.v17i0.247

www.criminologia.net

ISSN: 1696-9219 
pudo acreditarse una verdadera "sumisión química" a través de la administración encubierta de sustancias por terceros que causó a la víctima una situación incapacitante. A partir del análisis de los factores más comunes encontrados, y de la revisión de estudios previos, se proponen algunas medidas para mejorar la prevención y detección de esta modalidad delictiva.

Palabras clave: abuso sexual, delitos sexuales facilitados mediante el uso de sustancias psicoactivas, sumisión química, vulnerabilidad química, victimización sexual.

\section{ABSTRACT}

This article analyses the criminal phenomenology about Drug-Facilitated Sexual Assault (DFSA). Through a descriptive analysis of a sample of court decisions after criminal procedures followed in Spain between 1999 and 2016, we examine different variables available on the crime, the victim and the offender. From the sample analyzed, it was observed that in most cases (76\%), the aggressor took advantage of a situation of "chemical vulnerability" (DFSA opportunistic), where the victim herself consumed voluntarily toxic substances. Only in $24 \%$ of cases could a true "chemical submission" (DFSA proactive) be proved through the covert administration of substances by the offender or other person, causing the victim a disabling situation. From the analysis of the most common factors, and the review of previous studies, we proposed some measures to improve the prevention and detection of these crimes.

Keywords: sexual abuse, Drug-Facilitated Sexual Assault (DFSA), proactive DFSA, opportunistic DFSA, sexual victimization.

\section{Introducción}

El concepto Sumisión Química (SQ) puede definirse como la administración de sustancias psicoactivas a una persona, sin su consentimiento, con fines delictivos (Xifró et al. 2015), de forma que se pueda manipular su voluntad o modificar su comportamiento. En estas circunstancias, los efectos de la sustancia administrada pueden evitar que la víctima se encuentre en condiciones de prestar su consentimiento libremente, de acuerdo con la ley, o presentar resistencia a su atacante.

La denominación SQ procede de la expresión francesa soumission chimique. Para algunos autores, como Sánchez Pérez y Fombellida Velasco (2013), esta denominación presenta el inconveniente de utilizar el término "sumisión", en tanto que sugiere unas connotaciones sexuales que obligan a replantear su uso general por impropio. En inglés, para

Revista Española de Investigación Criminológica

Artículo 5, Número 17 (2019)

https://doi.org/10.46381/reic.v17i0.247

www.criminologia.net

ISSN: 1696-9219 
referirse al uso fraudulento de sustancias para cometer delitos la literatura especializada utiliza distintas denominaciones: drug-facilitated crime (DFC), o drug-facilitated assault $(D F A)$, que podrían traducirse de forma más fidedigna como "delito facilitado por sustancias” (DFS).

Se utiliza la SQ para facilitar la comisión de delitos tanto de naturaleza económica (sustracciones o delitos patrimoniales perpetrados mediante la influencia indebida, por ejemplo, en ancianos) como de carácter sexual, modalidad que tiene lugar en mayor proporción. En estos últimos, a pesar de que no es un fenómeno nuevo, desde finales de la década de los noventa del siglo pasado se ha observado en el contexto español un incremento del número de casos (García-Repetto \& Soria, 2011).

En el ámbito de los delitos sexuales y, en concreto, en los delitos de abuso sexual, se viene utilizando la denominación más específica "Drug-Facilitated Sexual Assault” (DFSA), refiriéndose a las relaciones sexuales no consentidas llevadas a cabo mientras la víctima se encuentra incapacitada o inconsciente, bajo los efectos de sustancias psicoactivas como alcohol, drogas ilícitas o psicofármacos (Shbair, 2010). La característica fundamental que distingue estos delitos de abuso sexual por uso de sustancias psicoactivas del resto de delitos de agresiones o abusos sexuales, es que la víctima sufre una pérdida de poder y control por el efecto producido por las sustancias psicoactivas consumidas, factor que es aprovechado por el agresor.

La literatura científica diferencia dos tipos de DFSA: (a) DFSA premeditado o proactivo, que tiene lugar cuando el asaltante proporciona a la víctima una sustancia incapacitante y desinhibidora con el objetivo de someterla sexualmente (le administra la sustancia sin su conocimiento, sin que pueda consentir); y (b) DFSA oportunista, que ocurre cuando el agresor se aprovecha de la víctima, hallándose ésta en estado de inconsciencia a causa de alguna sustancia que ha consumido voluntariamente. A esta segunda modalidad, también se le denomina vulnerabilidad química (VQ). En relación a esta distinción, también encontramos la denominación y diferenciación entre "date-rape" o violación en citas (DSFA oportunista) y "drug-rape", entendiéndose en este caso aquellas situaciones en que el ofensor 
droga a la víctima con la intención de violarla (DSFA premeditado) (Butler \& Welch, 2009; Gálvez, González \& Consuegra 2011).

Por otro lado, el Advisory Council on the Misuse of Drugs (UK) definió en 2007 la DFSA como todo acto en el que se realice una actividad sexual con una persona que sufre los efectos de drogas de abuso o medicamentos, y ello con independencia de que la administración haya sido forzada o voluntaria, o de que si la víctima hubiera consumido de forma voluntaria sustancias legales controladas. Esta definición incluye los dos tipos de DFSA, destacando que lo relevante es la afectación de la capacidad de consentimiento de la persona, sin diferenciar la voluntariedad o no del consumo o el tipo de sustancia consumida.

De manera análoga, la Agencia Francesa de Seguridad Sanitaria y Productos Sanitarios (AFSSAPS), establece tres categorías de SQ: (1) SQ probada: aquélla en la que ha habido agresión o tentativa documentada, se detecta la presencia de una sustancia psicoactiva mediante método cromatográfico fiable, y la sintomatología y cronografía de los hechos son compatibles con la sustancia identificada; (2) SQ probable: donde se sospecha que hubo agresión, pero no se dispone de suficiente información clínica o resultados analíticos, o no son éstos definitivos, o son negativos, o los análisis realizados son incompletos; y, (3) VQ, en la que la víctima reconoce el consumo voluntario de medicamentos, sustancias psicoactivas o ambas simultáneamente.

De las sustancias empleadas para dejar a la víctima en estado de inconsciencia o semiinconsciencia, la más preponderante y utilizada es el alcohol, sobre todo en los supuestos de VQ (DFSA oportunista) pero no es la única. En los casos de SQ propiamente (DFSA premeditado), las sustancias psicoactivas utilizadas reúnen una serie de características que las hacen adecuadas para el fin que persigue el agresor (Cruz Landeira, 2008): (i) son sustancias de acción rápida y de corta duración para, por un lado, facilitar el control sobre la víctima y, por otro lado, para no levantar sospechas; (ii) producen efectos que pueden llevar a confundir al clínico y retrasar el diagnóstico mientras la sustancia se elimina del organismo; (iii) son sustancias fáciles de obtener; (iv) la administración a la víctima debe ser discreta, habitualmente oral y añadidas en bebidas alcohólicas, siendo éstas el mejor vehículo para disimular el color y el sabor, así como potenciar sus efectos; (v) deben ser de difícil detección

Revista Española de Investigación Criminológica

Artículo 5, Número 17 (2019)

https://doi.org/10.46381/reic.v17i0.247

www.criminologia.net

ISSN: 1696-9219 
por la víctima; (vi) han de ser activas a dosis bajas; y (vii) deben producir los efectos buscados por el agresor: amnesia anterógrada, sedación, efectos alucinógenos y desinhibición. Como ha sido expuesto, aunque la más preponderante y utilizada ha sido el alcohol, no ha sido la única. En los últimos años se vienen utilizando otras sustancias psicoactivas como drogas ilegales o psicofármacos (por ejemplo, cocaína, ketamina, metanfetaminas, gammahidroxibutirato (GHB), benzodiacepinas o, algunos inhalantes volátiles) (Slaughter, 2000).

\section{Estado de la cuestión}

A pesar de que no es un fenómeno nuevo, se ha observado un incremento de casos en la década de los noventa (McGregor et al., 2004), lo que ha propiciado estudios en diversos países: por ejemplo, en Canadá (McGregor et al., 2004), Estados Unidos (Juhascik, Negrusz, Faugno \& Ledray, 2007), Polonia (Tomaszewska \& Krahé, 2015), Reino Unido (Gee, Owen, McLean, Brentha \& Thundercloud, 2006). En España, en el ámbito médico-forense, aunque se ha destacado la necesidad de conocer la magnitud de la SQ y las tendencias del fenómeno, existen pocos estudios epidemiológicos sobre la incidencia y prevalencia de este tipo de agresión (García-Caballero, Cruz-Landeira \& Quintela-Jorge, 2014; García-Repetto \& Soria, 2013; Navarro \& Vega, 2013; Xifró et al., 2014). De acuerdo con estos estudios, en los 179 casos de agresión sexual valorados por el Instituto de Medicina Legal de Alicante durante los años 2009-2012, en 19 casos (10.6\%) existió sospecha de SQ. En Barcelona, el estudio realizado por Arroyo et al., en 2011, de los 114 casos, 35 (30.70\%) cumplieron los criterios de SQ, En el estudio de García-Caballero, Cruz-Landeira y Quintela-Jorge (2014) realizado entre 2009 a 2012, de los 306 casos de agresión sexual que examinaron, el 35\% (107) fueron sospechosos de SQ.

Los estudios muestran que en casi la totalidad de los supuestos de SQ, las víctimas son mujeres (96.1\%) (Navarro \& Vega, 2013), 97\% (García-Caballero, Cruz-Landeira \& Quintela-Jorge, 2014), 100\% (García-Repetto \& Soria, 2011), 98.4\%, (Xifró et al.; 2015),

Revista Española de Investigación Criminológica

Artículo 5, Número 17 (2019)

https://doi.org/10.46381/reic.v17i0.247

www.criminologia.net

ISSN: 1696-9219 
91.4\% y de una media de edad de 25.3 años (Navarro y Vega (2013): 23 años; GarcíaCaballero, Cruz-Landeira y Quintela-Jorge (2014): 25.9 años y Xifró et al. (2015): 27 años).

Respecto al agresor, los estudios refieren que en la mayoría de casos es de sexo varón, que actúa solo y que, en el momento de la agresión, acababa de conocer a la víctima.

Coinciden los estudios en que, en la mayoría de casos los hechos ocurren en horas nocturnas, con prevalencia en los días de fin de semana, siempre en situaciones de ocio o actividades sociales, y con presencia de alcohol y consumo efectivo del mismo, solo o junto a otras sustancias. En el estudio de Xifró et al. (2015), en el 100\% de los casos se acreditó un consumo voluntario de alcohol por parte de la víctima durante o con antelación al concreto hecho delictivo. En el estudio de Navarro y Vega (2013), el 68\% de las víctimas refirieron un consumo previo de alcohol y en el de García Caballero, Cruz-Landeira, Quintela-Jorge (2014) en el 71\% de los casos se llegó a constatar dicho consumo.

Asimismo, en el estudio de Xifró et al. (2015) se determinaron cuatro factores idóneos para la concurrencia de esta tipología delictiva: (1) existencia de una actividad social previa con presencia de alcohol; (2) que dentro de esta actividad haya un consumo efectivo de alcohol por parte de la víctima; (3) que los presuntos hechos se comentan durante la noche; (4) y que el agresor sea una persona recién conocida para la víctima.

Desde el punto de vista de las teorías criminológicas, para comprender la dinámica de los delitos sexuales cometidos mediante el uso de sustancias psicoactivas deben tenerse en cuenta las premisas fundamentales de la teoría de la elección racional (Cornish \& Clarke, 2003), de las actividades cotidianas (Cohen \& Felson, 1979) y de los patrones delictivos (Brantingham \& Brantingham, 1995), todas ellas agrupadas bajo la denominación de las teorías de la oportunidad en el marco de la criminología ambiental. Dicho de otro modo, tales teorías presuponen de forma similar que la racionalidad del delincuente como hipótesis explicativa debe tener lugar necesariamente en un contexto, en un escenario espacio-temporal determinado (Agustina \& Reales, 2013). Así en la teoría del delito como elección racional (Cornish \& Clarke, 2003), el delito es producto de una elección racional tomada sobre la base de los costes y beneficios que una conducta puede proporcionarle al ofensor. De este modo, un individuo tenderá a decidir cometer un delito cuando, en un balance subjetivo global, los

Revista Española de Investigación Criminológica

Artículo 5, Número 17 (2019)

https://doi.org/10.46381/reic.v17i0.247

www.criminologia.net

ISSN: 1696-9219 
beneficios excedan a los costes. A este respecto, las 25 técnicas desarrolladas por Cornish y Clarke (2003) podrían ayudar en la motivación de potenciales agresores teniendo en cuenta: el fácil acceso a sustancias tóxicas, la situación de indefensión en que se encuentra la víctima por su consumo, así como los efectos amnésicos (amnesia anterógrada) que producen estas substancias y que hacen que el ofensor no pueda ser descubierto.

Estas teorías presuponen que la motivación del delincuente para cometer los delitos debe tener lugar necesariamente en un contexto, en un escenario espacio-temporal determinado. En estos casos, la probabilidad de que ocurra un hecho delictivo en un lugar y tiempo determinado se considera la función resultante de la convergencia de delincuentes racionales y motivados, objetivos adecuados y la ausencia de guardianes capaces (Cohen \& Felson, 1979). Es, en los delitos de abuso sexual mediante SQ, dentro del escenario espaciotemporal que llevaría a su comisión destacan factores como el estilo de ocio nocturno, tanto de las víctimas como de los agresores, las circunstancias facilitadoras de la agresión (como el fácil acceso a los tóxicos), la situación de inconsciencia o desinhibición de la víctima, y la tolerancia social frente a la promiscuidad, que explicaría que los "guardianes capaces" no actúen en defensa de situaciones de vulnerabilidad aparente de la víctima.

En este contexto, singular importancia merece el factor facilitador o precipitante del consumo del alcohol y drogas para la comisión de los delitos sexuales. Diversos estudios acreditan que el consumo de alcohol y drogas producen, en efecto, un incremento de la victimización sexual (Abbey, Zawacki, Buck, Clinton \& McAuslan, 2002; Testa \& Livingston, 2009), sin que por ello se deba culpabilizar a la mujer que consume alcohol o tóxicos, como causante de su victimización: Esta circunstancia no debe, en este sentido, generar duda alguna respecto a que es el agresor, casi siempre hombre, quien debe ser responsable criminal de toda agresión inconsentida, y respecto a que es necesario que la prevención se dirija a la perpetración masculina (Redondo \& Martínez Catena, 2012; Testa \& Livingston, 2009). Un claro ejemplo es el programa de prevención "No means no to yes means yes" establecido en los campus universitarios norteamericanos (New Jersey, California, New York, Connecticut, entre otros), con la finalidad de establecer un nuevo concepto de consentimiento en las relaciones sexuales ya que, en el año 2014, 55

\section{Revista Española de Investigación Criminológica}

Artículo 5, Número 17 (2019)

https://doi.org/10.46381/reic.v17i0.247

www.criminologia.net

ISSN: 1696-9219 
universidades norteamericanas fueron investigadas por la policía federal por supuestos de agresión sexual a mujeres universitarias (Frazer, 2014).

Obviamente la propia víctima, como estrategia de prevención, puede reducir las probabilidades de victimización cambiando aquellos comportamientos que hacen aumentar su vulnerabilidad, sin que ello implique responsabilidad en caso de sufrir una victimización (Igareda \& Boledón, 2014).

En relación a las estrategias de prevención dirigidas a las víctimas, debe señalarse que algunos estudios realizados en campus universitarios de Estados Unidos (Abbey et al, 2002; Ullman \& Brecklin, 2003) han constatado la correlación que existe entre el consumo voluntario en exceso de bebidas alcohólicas, denominado Heavy Episodic Drinking (HED), en las mujeres universitarias, y la situación de riesgo a ser víctimas de una agresión sexual. Desde esta perspectiva, la agresión sexual es considerada una de las consecuencias negativas atribuibles al HED. Y, por ello, en numerosos campus universitarios de Estados Unidos se realizan programas de intervención y prevención para reducir el alto consumo de alcohol de las estudiantes universitarias para reducir las tasas de victimización sexual (Testa \& Livingston, 2009).

Por otro lado, estudios como el de Igareda \& Boledón (2014) se plantea el establecimiento de protocolos de actuación en las universidades para los casos de violencia sexual, que incluirían no sólo orientación y asesoramiento, sino también derivación a recursos especializados para las posibles víctimas.

Debido al aumento de este fenómeno delictivo, en los últimos años se está produciendo en el ámbito español un auge en las investigaciones en el ámbito médicoforense. Pero se desconocen la existencia de revisiones específicas en curso en relación a esta modalidad delictiva desde el punto de vista propiamente criminológico. Por ello, mediante el presente estudio se pretende realizar un primer análisis criminológico, de carácter descriptivo-prospectivo, basado en una muestra de sentencias, sobre los diferentes factores ambientales de esta modalidad delictiva las características y perfil del agresor y de la víctima, y las drogas más utilizadas con este fin. 


\section{Objetivos e hipótesis}

El estudio que se presenta tiene como objetivo realizar un análisis descriptivo de la concurrencia de distintos factores o categorías en la muestra de sentencias obtenida. Concretamente se examinará distintos elementos que integran el modus operandi, así como aquellos que pueden contribuir a configurar un perfil de agresor y de víctima.

En base a todo ello, se formularon las siguientes hipótesis:

a. La vulnerabilidad química se presenta en la mayoría de casos con respecto a las situaciones de sumisión química propiamente dicha, y ello es debido a la alta concurrencia de alcohol, que es consumido por la propia víctima, coincidiendo con los estudios realizados por Cruz-Landeira, et al. (2008) o Isorna y Rial (2015).

b. Los hechos se producen durante fin de semana y en horas nocturnas, normalmente en situaciones de ocio, de acuerdo con los estudios de Xifró et al. (2015), Navarro y Vega (2013), o el realizado por García-Caballero, Cruz-Landeira y Quintela-Jorge (2014).

c. La víctima es una mujer, joven, de acuerdo con los estudios de Mc Gregor et al. (2004); Testa \& Livingston, (2009); Navarro y Vega, (2013), García Caballero, CruzLandeira y Quintela-Jorge, (2014); García-Repetto y Soria, (2013); Xifró et al., (2015); Isorna y Rial, (2015) de nacionalidad española y el agresor es un varón, de acuerdo con el estudio realizado por Cruz-Landeira et al. (2008). Y que existe una relación previa entre víctima y agresor; bien es un amigo, expareja, vecino o conocido reciente, y sólo en un número reducido de casos el agresor es un completo desconocido (Cruz Landeira, et al., 2008).

\section{Metodología}

Para lograr los fines que se proponía la presente investigación, se llevó a cabo una búsqueda en las bases de datos jurisprudenciales CENDOJ (Centro de Documentación Judicial del Consejo General del Poder Judicial) y Aranzadi Instituciones, utilizando determinadas

Revista Española de Investigación Criminológica

Artículo 5, Número 17 (2019)

https://doi.org/10.46381/reic.v17i0.247

www.criminologia.net

ISSN: 1696-9219 
palabras clave, obteniendo 102 sentencias que enjuiciaban hechos delictivos cometidos entre 1999 y $2016^{2}$.

De las sentencias obtenidas, se hizo una primera clasificación entre sentencias donde el abuso sexual se había cometido de forma oportunista (DFSA oportunista) y aquéllas en que el hecho se cometió de forma premeditada (DFSA premeditada). Dentro de cada grupo de sentencias se hizo una discriminación entre sentencias absolutorias y condenatorias, descartando las sentencias absolutorias, y centrando el análisis solo en las condenatorias, en las que queda demostrada la concurrencia del delito de abuso sexual mediante el uso de sustancias psicoactivas. Con ello se pretendió eliminar los posibles falsos positivos que podría haber. Pero, en todo caso, se debe tener en cuenta que existe una importante "cifra negra" (diferencia entre la criminalidad aparente y la real) en estos delitos: por un lado, existe la posibilidad de que muchas sentencias sean absolutorias porque no se ha llegado a demostrar en el proceso penal, por falta de pruebas, la existencia del delito y, por otro lado, como es sabido no todos los hechos son denunciados por las víctimas (Garrido, Stangeland \& Redondo, 2006). Realmente existe una distancia significativa entre la gran magnitud de los episodios de abuso y agresiones sexuales que realmente suceden y aquellos que llegan a conocerse y denunciarse formalmente (Martínez Catena \& Redondo, 2016).

Respecto a las sentencias condenatorias se realizó un análisis de las mismas recogiendo las siguientes variables:

1.- Tipo de sustancia utilizada. 2.- Tipo de prueba para la detección de tóxicos (solo en el abuso sexual cometido de manera premeditada). 3.- Lugar donde ocurrieron los hechos. 4.- Día de la semana en que tuvieron lugar los hechos. 5.- Estación del año. 6.- Momento del día en que sucedieron los hechos. 7.- Número de agresores en los hechos. 8.- Sexo de los agresores. 9.- Edad. 10.- Nacionalidad. 11.- Antecedentes Penales. 12.- Número de víctimas

\footnotetext{
${ }^{2}$ Las palabras clave utilizadas han sido: "abuso sexual anulando la voluntad de la víctima por consumo de drogas"; "abuso sexual y alterar la voluntad de la víctima mediante sustancias"; "abuso sexual y benzodiacepina"; "abuso sexual y burundanga"; "abuso sexual y escopolamina"; "abuso sexual y sustancias químicas"; "art. 181.2 CP y anulando la voluntad de la víctima mediante sustancias tóxicas"; "sumisión química".
}

Revista Española de Investigación Criminológica

Artículo 5, Número 17 (2019)

https://doi.org/10.46381/reic.v17i0.247

www.criminologia.net

ISSN: 1696-9219 
en cada hecho. 13.- Sexo de la víctima. 14.- Edad. 15.- Nacionalidad. 16.- Relación entre víctima y agresor.

Los datos fueron tratados con Microsoft Excel (Microsoft Office Profesional Plus 2016, versión 1701), para su posterior tratamiento estadístico con el software SPSS 21.0. Para determinar las características de la muestra se empleó un análisis descriptivo (distribución de frecuencias absolutas y porcentajes válidos, media y desviación estándar). Asimismo, se llevó a cabo un análisis comparativo utilizando la prueba chi-cuadrado ( $\chi 2)$ para variables cualitativas, con la finalidad de analizar la posible relación entre algunas de las variables y el comportamiento delictivo. Sin embargo, debido al relativamente pequeño tamaño muestral del estudio, el análisis comparativo no ha arrojado resultados estadísticamente significativos.

\section{Resultados}

Se obtuvo una muestra de 102 sentencias, en las que se recogían los supuestos de DFSA premeditado (SQ) o DFSA oportunista (VQ) cometidos entre 1999 y 2016. De éstas, 95 fueron dictadas por Audiencias Provinciales, 1 por la Audiencia Nacional, y 6 por el Tribunal Supremo.

De estas 102 sentencias se hizo una primera discriminación entre sentencias absolutorias y condenatorias, obteniendo 27 sentencias absolutorias y 75 condenatorias. Para el estudio, se han descartado las sentencias absolutorias y solo se han analizado las condenatorias, en tanto que, como se ha señalado, son aquellas en las que queda acreditada la existencia de abuso sexual mediante SQ o VQ.

Tipo de abuso y sustancia

En la Tabla 1 se muestra la prevalencia de casos de vulnerabilidad química (VQ) sobre aquellos en los que concurrieron situaciones de sumisión química (SQ): de las 75 sentencias condenatorias, 54 fueron casos de VQ y 21 de SQ. Esta prevalencia de la VQ, que es aquella donde la propia víctima se sitúa en situación de inconsciencia, es debida a la alta concurrencia de alcohol, como se puede observar en la tabla. En el 94.5\% de los casos de vulnerabilidad

Revista Española de Investigación Criminológica

Artículo 5, Número 17 (2019)

https://doi.org/10.46381/reic.v17i0.247

www.criminologia.net

ISSN: 1696-9219 
química se constata consumo de bebidas alcohólicas, ya sea de manera individual o junto a otro tóxico como drogas o fármacos. En cambio, en los casos de SQ también se observa la prevalencia del alcohol, pero, en este caso, no de forma individual sino junto a fármacos o drogas, siendo su conjunción el porcentaje más elevado (alcohol con fármacos el 47.6\% $(n=10)$, y alcohol con drogas el 38.1\% $(n=8))$.

\section{Tabla 1.}

Tipo de abuso (VQ y SQ) y sustancias

\begin{tabular}{|c|c|c|c|c|}
\hline & \multicolumn{2}{|c|}{ VQ } & \multicolumn{2}{|c|}{ SQ } \\
\hline & $\begin{array}{c}\text { Frecuencia } \\
\text { Absoluta }\end{array}$ & \% Válido & $\begin{array}{c}\text { Frecuencia } \\
\text { Absoluta }\end{array}$ & \% Válido \\
\hline Tipo de abuso & 54 & $72 \%$ & 21 & $28 \%$ \\
\hline \multicolumn{5}{|l|}{ Sustancia } \\
\hline Alcohol & 36 & $66.7 \%$ & 0 & $0.0 \%$ \\
\hline Alcohol + Fármacos & 2 & $3.7 \%$ & 10 & $47.6 \%$ \\
\hline Alcohol + Drogas & 13 & $24.1 \%$ & 8 & $38.1 \%$ \\
\hline Fármacos & 2 & $3.7 \%$ & 2 & $9.5 \%$ \\
\hline Drogas & 1 & $1.9 \%$ & 1 & $4.8 \%$ \\
\hline
\end{tabular}

En relación a las sustancias tóxicas, se ha comprobado en las sentencias si en ellas queda plasmada la práctica de alguna prueba de detección de tóxicos. Hay que destacar que en el $80 \%$ de los casos $(n=60)$ no hay constancia de que se practicara ningún tipo de prueba para la detección del tóxico. En el resto de casos, la más practicada es la prueba conjunta de sangre y orina, representado en el 8\% $(n=6)$ de los casos; seguidamente consta en un 5\% $(n=4)$ de sentencias que se practicó de manera individualizada prueba de sangre o prueba de orina; y, finalmente, solo en un caso consta que se le hiciera a la víctima la prueba de detección de tóxicos mediante una muestra de su cabello.

Respecto al hecho delictivo, el 76\% $(\mathrm{n}=57)$ de los abusos mediante SQ/VQ terminan en penetración, mientras que en el $24 \%$ de casos $(n=18)$ los abusos consisten en tocamientos del agresor a la víctima.

La exposición de los siguientes resultados no se hace de manera diferenciada en función de $\mathrm{VQ} / \mathrm{SQ}$, sino que se presentan de manera conjunta dado que los dos grupos no presentan diferencias en los resultados obtenidos. 


\section{Elemento temporal}

En cuanto al elemento temporal, se observa que en el 30.7\% $(n=23)$ de los casos ocurren en primavera, seguido de la época de verano $(28 \%(n=21))$ e invierno $(26.7 \%)$. Respecto al día de la semana en que acontecen los hechos, se observa que la mayoría de ellos se comenten en fin de semana (de viernes a domingo), destacando sábado en el $28 \%$ de los casos (n=21). Y en cuanto al momento del día, ${ }^{3}$ en la mayoría de los casos los hechos ocurren en horas nocturnas, llegando hasta el $65.3 \%$ de los casos analizados.

\section{Tabla 2.}

Elemento temporal

\begin{tabular}{lcc}
\hline & Frecuencia Absoluta (n) & \% Válido \\
\hline Época del año & & \\
\hline Primavera & 23 & $30.7 \%$ \\
Verano & 21 & $28.0 \%$ \\
Otoño & 8 & $10.7 \%$ \\
Invierno & 20 & $26.7 \%$ \\
No consta & 3 & $4.0 \%$ \\
\hline Día de la semana & & \\
\hline Lunes & 4 & $5.3 \%$ \\
Martes & 9 & $12.0 \%$ \\
Miércoles & 5 & $6.7 \%$ \\
Jueves & 5 & $6.7 \%$ \\
Viernes & 11 & $14.7 \%$ \\
Sábado & 21 & $28.0 \%$ \\
Domingo & 15 & $20.0 \%$ \\
No especificado & 5 & $6.7 \%$ \\
\hline Momento del día & & \\
\hline Mañana & 2 & $2.7 \%$ \\
Tarde & 18 & $24.0 \%$ \\
Noche-madrugada & 49 & $65.3 \%$ \\
No consta & 5 & $6.7 \%$ \\
Continuado & 1 & $1.3 \%$ \\
\hline
\end{tabular}

\section{Elemento espacial}

En cuanto al lugar donde se comete el hecho delictivo, hay una prevalencia de lugares privados (64\%) frente a espacios públicos (33.3\%); destacando en primer lugar el domicilio

\footnotetext{
${ }^{3}$ En referencia a la distribución del momento del día: mañana, de las $6 \mathrm{~h}$ a las $14 \mathrm{~h}$; tarde, de las $14 \mathrm{~h}$ a las $22 \mathrm{~h}$; noche de las $22 \mathrm{~h}$ a las $6 \mathrm{~h}$.
}

Revista Española de Investigación Criminológica

Artículo 5, Número 17 (2019)

https://doi.org/10.46381/reic.v17i0.247

www.criminologia.net

ISSN: 1696-9219 
del ofensor (en un 33.3\% de los casos), seguido del domicilio de la víctima (12\%), del de un amigo $(6.7 \%)$ o en un coche (12\%). Respecto a los delitos cometidos en espacios públicos destaca que el $26.7 \%$ de los mismos se cometieron en la vía pública (calles o parques).

\section{Tabla 3.}

Lugar donde ocurren los hechos

\begin{tabular}{lcc}
\hline & Frecuencia (n) & Porcentaje (\%) \\
\hline Domicilio Agresor & 25 & $33.3 \%$ \\
Domicilio Víctima & 9 & $12.0 \%$ \\
Vía pública & 20 & $26.7 \%$ \\
Bar - discoteca & 4 & $5.3 \%$ \\
Coche & 9 & $12.0 \%$ \\
Domicilio amigo & 5 & $6.7 \%$ \\
Cajero automático & 1 & $1.3 \%$ \\
No consta & 2 & $2.7 \%$ \\
\hline
\end{tabular}

Concurrencia de agresores y de víctimas

En cuanto al número de agresores, de los 75 casos analizados, en el $86.7 \%(\mathrm{n}=65)$ son cometidos por un solo agresor, mientras que en el 12\% de los casos $(n=9)$ intervienen dos agresores y solo en un caso (1.3\%) concurren tres ofensores.

Respecto al número de víctimas, de las 75 sentencias analizadas, en el 82\% de ellas $(n=69)$ el delito se cometió sobre una sola víctima, mientras que, en un porcentaje menor, en el $8 \%(n=6)$ el número de víctimas fueron dos.

En cuanto a la relación previa con la víctima, en un $72 \%$ de casos $(n=54)$ el acusado era una persona conocida por la víctima, mientras que en un $28 \%(n=21)$ se trataba de un completo desconocido. Dentro de los casos en que existía un previo conocimiento entre víctima y agresor, en un $40.7 \%$ de los casos son conocidos, mientras que en un $38.8 \%$ de casos existía una relación de amistad; y en un $14.8 \%$ de casos $(n=8)$ había una relación de parentesco entre agresor y víctima. 


\subsection{Perfil del agresor}

De las sentencias analizadas se han podido extraer algunas variables que pueden conformar un perfil de agresor. El análisis se realiza con una muestra de 85 agresores cuyos resultados se pueden observar en la tabla 4.

La media de edad del ofensor es de 32.7 años, siendo la edad mínima de 15 años, la máxima de 57 años, y la moda de 19. En el 98.8\% de los casos el ofensor es de sexo masculino: solamente existe un caso en que es mujer, actuando en el mismo no como autora directa sino como cooperadora necesaria.

En referencia a la nacionalidad, en el $44 \%$ de los casos $(n=38)$ el agresor es de nacionalidad española, mientras que el 35.3\% es de nacionalidad extranjera. No obstante, debe tenerse en cuenta que en 17 casos (20\% de la muestra), donde no se indica en la sentencia la nacionalidad del agresor.

\section{Tabla 4.}

Perfil del agresor

\begin{tabular}{lcc}
\hline & Frecuencia (n) & Porcentaje (\%) \\
\hline Sexo & & \\
\hline Hombre & 84 & $98.8 \%$ \\
Mujer & 1 & $1.2 \%$ \\
\hline Nacionalidad & & $44.7 \%$ \\
\hline Española & 38 & $35.3 \%$ \\
Extranjera & 30 & $20.0 \%$ \\
No consta & 17 & $81.2 \%$ \\
\hline Antecedentes Penales & & $8.2 \%$ \\
\hline No & 69 & $10.6 \%$ \\
Si & 7 & \\
No consta & 9 & \\
\hline
\end{tabular}




\subsection{Perfil de la víctima}

De las sentencias analizadas se han podido obtener las siguientes variables que permiten conformar un perfil de víctima. El análisis se realiza con una muestra de 81 víctimas, cuyos resultados se muestran en la Tabla 5.

La media de edad de la víctima es de 19.2 años, siendo la mínima de 10 años y la máxima de 47. Aunque este dato no es del todo preciso ya que, de las 81 víctimas, de solo 34 de ellas se determina su edad, habiendo 47 casos perdidos.

En referencia al sexo de la víctima, en el $96.3 \%$ de los casos, se trata una mujer, mientras que en el 3.7\% $(n=3)$ es un hombre. Y en cuanto a la nacionalidad, solo está determinada en 49 víctimas, (60.4\%), habiendo 32 casos donde no consta su nacionalidad. En los casos en que sí se determina, se observa que en la mayoría de los mismos es de nacionalidad extranjera.

\section{Tabla 5.}

Perfil de la víctima

\begin{tabular}{lcc}
\hline & Frecuencia (n) & Porcentaje (\%) \\
\hline Sexo & & \\
\hline Mujer & 78 & $96.3 \%$ \\
Hombre & 3 & $3.7 \%$ \\
\hline Nacionalidad & & \\
\hline Española & 13 & $16.0 \%$ \\
Extranjera & 36 & $44.4 \%$ \\
No consta & 32 & $39.5 \%$ \\
\hline
\end{tabular}

\section{Discusión}

Con el presente estudio se ha realizado una aproximación a los elementos descriptivos de los denominados DFSA. Tras el análisis empírico realizado a partir de una muestra de sentencias condenatorias $(\mathrm{N}=75)$, se ha podido observar que existe una prevalencia de casos de DFSA oportunista (VQ) frente a la victimización derivada de DSFA premeditada (SQ). El motivo

\section{Revista Española de Investigación Criminológica}


de esta diferencia cuantitativa es debido a que la detección de los casos de DFSA de tipo premeditado se ve dificultada por varios factores: por un lado, el retraso en solicitar ayuda por parte de la víctima facilita la rápida eliminación del tóxico; también la baja dosis del mismo, así como la fácil confusión de este cuadro clínico con el de una intoxicación etílica voluntaria, ya que, por el momento, la toxicología no puede discernir el consumo voluntario del involuntario, sobre todo cuando la sustancia causante del cuadro es asiduamente consumida por la propia víctima (Cruz-Landeira et al., 2008; Isorna \& Rial, 2015). Además, la amnesia anterógrada es uno de los efectos secundarios de estas sustancias utilizadas para cometer estos delitos que, junto al estado de aturdimiento en que se encuentra la víctima en ese momento, hace que ésta no pueda recordar nada sobre los hechos (solo puede recordar escenas) ni tampoco identificar a su agresor. Por ello, muchos delitos no llegan a ser denunciados, resultando difícil evaluar la incidencia real del delito de abuso sexual mediante DFSA premeditada (Elliot, 2008, Isorna \& Rial, 2015; Saint-Martin, Bouyssy \& O’Byrne, 2007).

La sustancia tóxica que prevalece en esta modalidad delictiva es el alcohol, bien de manera individual, como es en la mayoría de casos de VQ, bien en concurrencia con drogas o fármacos, más común en los casos de SQ.

Los resultados obtenidos sugieren que, con carácter general, se trata de delitos que suelen ocurrir mayoritariamente en las horas nocturnas, con prevalencia en los días de fin de semana (desde el viernes hasta el domingo), coincidiendo así con los estudios realizados por Xifró et al. (2015), Navarro y Vega (2013), García-Caballero, Cruz-Landeira y QuintelaJorge (2014). En todos ellos se apunta que se trata de hechos cometidos en situaciones de ocio nocturno y con prevalencia en los fines de semana. Respecto a la época del año en que ocurren los hechos, la mayoría de ellos se cometen en primavera, si bien también hay una alta prevalencia de delitos cometidos en verano e invierno.

Es destacable, así mismo, las pocas pruebas que se les practican a las víctimas para la detección de tóxicos. De los casos en que se deja constancia de esta circunstancia, en la mayoría de ellos se hace una prueba de sangre y orina conjuntamente, aunque muchas veces resulta ser insuficiente debido al transcurso de tiempo que pasa entre los hechos ocurridos y

Revista Española de Investigación Criminológica

Artículo 5, Número 17 (2019)

https://doi.org/10.46381/reic.v17i0.247

www.criminologia.net

ISSN: 1696-9219 
la toma de muestras: según algunos estudios científicos el tiempo trascurrido es de 18.26 horas (Escayola \& Vega, 2013), lapso temporal en el que el tóxico ya ha podido ser eliminado del cuerpo de la víctima. Por eso, sería conveniente que el personal de los servicios de urgencias que atienden a las víctimas de este tipo de delitos pueda conocer un protocolo de actuación en estos casos, ya que es importante observar un cuidadoso examen físico, así como practicar la toma y conservación de las muestras biológicas obtenidas. A este respecto, la literatura médico-forense recomienda obtener muestras tanto de sangre como de orina dentro de las primeras 48 horas desde los hechos: entre las 48 y las 168 horas (7 días) se recomienda obtener solo muestra de orina. Pasados los 7 días tras los hechos se recomienda realizar un análisis del cabello (Isorna \& Rial, 2015; Xifró, et al., 2015).

Respecto al perfil de víctima, en la mayoría de los casos hay una prevalencia del sexo femenino, coincidiendo con otros autores (García Caballero, Cruz-Landeira \& QuintelaJorge, 2014; García-Repetto \& Soria, 2013; Isorna \& Rial, 2015; McGregor et al. 2004; Navarro \& Vega, 2013; Testa \& Livingston, 2009; Xifró et al., 2015). Con el análisis jurisprudencial realizado no se pueden determinar otros factores de suficiente entidad, debido a que en la mayoría de las sentencias se omite mucha información relativa a las víctimas, como la edad o la nacionalidad, variables que hemos tratado de recoger y analizar en este estudio. Para futuras investigaciones se recomienda elaborar un protocolo de recogida de información con vistas a construir de forma más completa un perfil de víctima.

En referencia al perfil del agresor, en la mayoría de los casos estos delitos son cometidos por varones, con independencia de que la víctima sea mujer u hombre. Solo se ha observado un único caso donde uno de los agresores es de sexo femenino, si bien actuando éste como cooperador necesario. Existe una prevalencia de la nacionalidad española del agresor y de ausencia de antecedentes penales.

En gran parte de los casos, existe algún tipo de vínculo previo entre víctima y agresor (ya sea un simple conocido, o medie una relación amistad, sentimental, e incluso familiar). En pocas ocasiones es un completo desconocido, tal y como se señala por otros autores (CruzLandeira, Quintela-Jorge \& López Rivadulla, 2008; Navarro \& Vega, 2013; Xifró et al., 2015).

Revista Española de Investigación Criminológica

Artículo 5, Número 17 (2019)

https://doi.org/10.46381/reic.v17i0.247

www.criminologia.net

ISSN: 1696-9219 
Atendiendo a los resultados obtenidos, se observa que en muchos casos no es el agresor el que administra a la víctima alguna sustancia tóxica sin su conocimiento, sino que es la propia víctima la que conscientemente se pone en situación de vulnerabilidad, por el consumo voluntario de alcohol, drogas, fármacos, o la mezcla de ellos aprovechándose el ofensor de esta situación para agredirla sexualmente. Nos encontramos, por un lado, ante un problema social donde existe una desigualdad de género, en la que la mujer es objeto de deseo sexual responsabilizando muchas veces a la propia víctima de haber generado la situación. Por otro lado, también puede enfocar la prevención de esta modalidad de victimización como un problema de salud pública, por el consumo, voluntario o no, de sustancias.

Como aportación relevante, las conclusiones expuestas permiten un análisis preliminar del perfil criminológico de los agresores, las víctimas y el modus operandi del hecho delictivo en relación al fenómeno de la SQ/VQ. Ante el reto planteado, el conocimiento de dicho perfil sienta las bases para establecer medidas preventivas ante esta situación. Varios estudios, como el de McGregor et al. (2004) y Elliot (2008), reflejan la necesidad de trabajar en prevención e información de potenciales víctimas, como son las chicas jóvenes menores de 20 años, las cuales son las más vulnerables a esta forma de delincuencia sexual, por lo que es importante crear programas de prevención específicos dirigido a este grupo de población.

\section{Limitaciones}

Desde un punto de vista metodológico, las conclusiones de este estudio deberían ser consideradas con cautela atendiendo a que la muestra de sentencias es relativamente pequeña, y a que se han excluido las sentencias absolutorias, por su menor interés en relación al objetivo planteado en este trabajo. Con todo, debe recordarse que, de acuerdo con la literatura criminológica (Martínez Catena \& Redondo, 2016), la mayoría de los casos de asalto sexual no se denuncian. 


\section{Referencias}

Abbey, A., Zawacki, T., Buck, P. O., Clinton, A. M., \& McAuslan, P. (2002). Alcohol and sexual assault. Alcohol Research \& Health, 25(1), pp 43-51

Agustina, J. R., \& Reales, F. (2013). En la mente de un asaltante de viviendas: Estudio cualitativo de una muestra de autores de robo en casa habitada. Revista Española de Investigación Criminológica, 11, 1-30.

Arroyo A, Barbería E, Bertomeu A, Montero F, Pujol A, Xifró A. (2011) Incidència i característiques de la submissió química en els delictes d'agressió sexual. Centre d'Estudis Jurídics i Formació Especialitzada: Barcelona. Disponible en: http://www20.gencat.cat/portal/site/Justicia

Brantingham P. \& Brantingham P. (1995). Criminality of place: Crime generators and crime attractors. European Journal on Criminal Policy and Research (3), pp. 5-26.

Butler, B. \& Welch, J. (2009). Drug-facilitated sexual assault. CMAJ, 5, pp.493-494. doi: $\underline{10.1503 / \mathrm{cmaj} .090006}$

Cohen, L. E. \& Felson, M. (1979). Social Change and Crime Rate Trends: A Routine Activity Approach. American Sociological Review 44 (4), pp. 588-608. Doi: $10.2307 / 2094589$

Cornish, D.B. \& Clarke, R.V. (2003). Opportunities, precipitators and criminal decisions: A reply to Wortley's critique of situational crime prevention. In Smith, M. and Cornish, D. B. (eds) Theory for Situational Crime Prevention, Crime Prevention Studies, 16, pp. 41-96..

Cruz-Landeira, A., Quintela-Jorge, O., López-Rivadulla, M. (2008). Sumisión química: epidemiología y claves para su diagnóstico. Medicina Clínica, 131(20), pp. 783-789.

Elliott, S. (2008). Drug-facilitated sexual assault: educating women about the risks. Nurs Womens Health, 12, pp.30-37. Doi: 10.1111/j.1751-486X.2007.00273.x

Escayola, E., Vega, C. (2013). Agresiones sexuales facilitadas por sustancias psicoactivas, detectadas en el Instituto de Medicina Legal de Alicante en el cuatrienio 2009-2012. Gaceta Internacional Ciencias Forense, 8, pp. 8-15. Recuperado de: https://www.uv.es/GICF/4A1_Navarro_GICF_08.pdf

Frazer, L. (october 8, 2014). From "no means no" to "yes means yes". 2017, de Berkely Political Rewiew Sitio web: https://bpr.berkeley.edu/2014/10/08/from-no-means-noto-yes-means-yes-why-sb-967-challenges-rape-culture/

Gálvez, E., González, Y.A., y Consuegra, E. (2011). Sexualidad y drogas: aspectos medicolegales de interés para el médico general integral. Revista Habanera de Ciencias Médicas, 10(4), pp. 492-495. Recuperado de: http://scielo.sld.cu/scielo.php?script $=$ sci arttext\&pid $=$ S1729$\underline{\text { 519X2011000400011\&lng=es\&tlng=es }}$

Revista Española de Investigación Criminológica

Artículo 5, Número 17 (2019)

https://doi.org/10.46381/reic.v17i0.247

www.criminologia.net

ISSN: 1696-9219 
García-Caballero, C., Cruz-Landeira, A. \& Quintela-Jorge, O. (2014). Sumisión química en casos de presuntos delitos contra la libertad sexual analizados en el Instituto Nacional de Toxicología y Ciencias Forenses (Departamento de Madrid) durante los años 2010, 2011 y 2012. Rev Esp Med Legal, 40, pp.11-18. doi: 10.1016/j.reml.2013.07.003

García-Repetto, R. \& Soria, M.L. (2011). Sumisión química: reto para el toxicólogo forense. Revista Española de Medicina Legal, 37, pp. 105-112. Doi: 10.1016/S03774732(11)70072-4

García-Repetto, R. \& Soria, M.L. (2013). Consideraciones toxicológicas sobre supuestos casos de sumisión química en delitos de índole sexual en el sur de España entre los años 2010-2012. Revista Española de Medicina Legal, 40 (1), pp. 4-10.

Garrido, V., Stangeland, P. \& Redondo, S. (2006). Principios de Criminología. Valencia: Tirant Lo Blanch.

Gee D, Owen P, McLean I, Brentha, K., Thundercloud, C. (2006). Operation MATISSE: investigating drug facilitated sexual assault. London (UK): The Association of Chief Police Officers (ACPO); disponible en: www.acpo.police.uk/asp/policies/Data/Operatin\%20Matisse\%20report\%20\%20press\%20rel.\%2084.doc

Igareda, N., Boledón, E. (2014). Las violencias sexuales en las universidades: cuando lo que no se denuncia no existe. Revista Española de Investigación Criminológica, 12 (1), pp. 1-27.

Isorna-Folgar, M., Fariña-Rivera, F., Sierra, J.C., Vallejo-Medina, C. (2015, Enero-Junio). Binge drinking: conductas sexuales de riesgo y drogas facilitadoras del asalto sexual en jóvenes españoles. Sum Psic, 22, pp. 1-8. Recuperado de doi: 10.1016/j.sumpsi.2015.05.001

Isorna-Folgar, M. \& Rial, A. (2015). Drogas facilitadoras de asalto sexual y sumisión química. Health and Addictions, 15 (2), pp.137-150. Disponible en: http://www.haaj.org/index.php/haaj/article/view/246/254

Juhascik, MP., Negrusz, A., Faugno, D., Ledray, L. (2007) An estimate of the proportion of the drug-facilitation of sexual assault in four U.S. localitates. J Forensic Sci., 52, pp. 1396-1400. Recuperado de doi: 10.1111/j.1556-4029.2007.00583.x

Martínez-Catena, A. \& Redondo, S. (2016) Etiología, prevención y tratamiento de la delincuencia sexual. Anuario de Psicología Jurídica, 26, pp. 19-29. Recuperado de doi: 10.1016/j.apj.2016.04.003

McGregor, M.J., Janssen, P., Ericksen, J., van Vliet, A., Ronald, L. \& Schulzer, M. (2004). Rising incidence of hospital-reported drug-facilitated sexual assault in a large urban community in Canada: Retrospective population-based study. Can J Public Health, 95, pp. 441-445. DOI: http://dx.doi.org/10.17269/cjph.95.557

Revista Española de Investigación Criminológica

Artículo 5, Número 17 (2019)

https://doi.org/10.46381/reic.v17i0.247

www.criminologia.net

ISSN: 1696-9219 
Navarro, E., Vega, C. (2013). Agresiones sexuales facilitadas por sustancias psicoactivas detectadas en el Instituto de Medicina Legal de Alicante en el cuatrienio 2009-2012. Gaceta Internacional de Ciencias Forenses, 8, pp 8-15.

Redondo, S. \& Martínez Catena, A. (2011). Tratamiento y cambio terapéutico en agresores sexuales. Revista Española de Investigación Criminológica, 9 (8), pp. 1-25.

Shbair, M.K., Lhermitte, M. (2010). Drug-facilitated crimes: Definitions, prevalence, difficulties and recommendations. A review. Annales Pharmaceutiques Francaises, 68 (3), pp. 136-147. doi: 10.1016/j.pharma.2010.03.005.

Saint-Martin, P. Bouyssy, M. \&O’Byrne, P. (2007). Analysis of 756 cases of sexual assault in Tours (France): medico-legal findings and judicial outcomes. Medicine, Science and the Law, 47, pp. 315-324. DOI: 10.1258/rsmmsl.47.4.315

Sánchez-Perez, J.D. \& Fombellida-Velasco, L. (2013). Consideraciones sobre el delito sexual facilitado por sustancias. Diario La Ley, 8054, pp. 1-4.

Testa, M. \& Livingston, J. (2009). Alcohol consumption and women's vulnerability to sexual victimization: can reducing women's drinking prevent rape? Subst Use Misuse, 44, pp.1349-1376. DOI: 10.1080/10826080902961468

Tomaszewska, P. \& Krahé, B. (2015, October). Sexual Aggression victimization and perpetration among female and male university students in Poland. Journal Interpersonal Violence, 33, pp.571-594. Recuperado de doi: https://doi.org/10.1177/0886260515609583.

Ullman, S.E. \& Brecklin, L.R. (2003). Sexual Assault History and Health-Related Outcomes in a National Sample of Women. Psychology of Women Quarterly 27(1), pp. 46-57. doi: 10.1111/1471-6402.t01-2-00006

Xifró-Collsamata, A. (2008, Enero). Drug facilitated sexual assault. Revista Española de Medicina Legal, 34, p.52. Recuperado de doi: 10.1016/S0377-4732(08)70028-2

Xifró-Collsamata, A., Pujol-Robinat, A., Barbería-Marcalain, E., Arroyo-Fernández, A., Bertomeu-Ruiz, A, Montero-Núñez, F. \& Medallo-Muñiz, J. (2015). Estudio prospectivo de la sumisión química con finalidad sexual en Barcelona. Revista Española de Medicina Legal, 144, pp. 403-409. Disponible en: http://dx.doi.org/10.1016/j.medcli.2014.11.026

\section{Agradecimientos}

Este trabajo ha sido desarrollado en el marco del proyecto de investigación en el que participa como miembro investigador el Dr. Agustina, y que lleva por título "Criminología, evidencias empíricas y política criminal. Sobre la incorporación de datos científicos para la toma de decisiones en relación con la criminalización de conductas" (Ref. DER2017-86204-R), financiado por la Agencia Estatal de Investigación (AEI) del Ministerio de Ciencia, Innovación y Universidades. 
Maria Neus Panyella-Carbó, Letrada de la Generalitat de Catalunya. Licenciada en Derecho por la Universitat de Barcelona y Máster en Psicopatología Legal, Forense y Criminológica por la Universidad Internacional de Catalunya. Actualmente se halla realizando su tesis doctoral que versa sobre el análisis jurídico-criminológico del uso de sustancia psicoactivas en los delitos sexuales.

José R. Agustina, Catedrático acreditado de Derecho penal en la Universitat Internacional de Catalunya y codirector del Máster de Psicopatología Legal, Forense y Criminológica de la Universitat Internacional de Catalunya.

https://orcid.org/0000-0002-9254-6902

Carles Martin-Fumadó, Doctor en Medicina. Médico forense en excedencia. Docente del Máster de Psicopatología Legal, Forense y Criminológica y del Grado en Medicina de la Universitat Internacional de Catalunya. Colabora en la Cátedra de Responsabilidad Profesional Médica y Medicina Legal del Departamento de Psiquiatría y Medicina Legal de la Universitat Autònoma de Barcelona.

https://orcid.org/0000-0003-2375-8423 